\title{
Elastic Metamaterials Making Use of Chirality: A Review
}

\author{
Xiaoning Liu - Gengkai Hu \\ Key Laboratory of Dynamics and Control of Flight Vehicle, Ministry of Education, \\ School of Aerospace Engineering, Beijing Institute of Technology, China
}

\begin{abstract}
Metamaterials are artificially designed composite materials exhibiting unusual physical properties not easily found in nature. In most cases, these properties appear in a wave environment where local resonance comes into play. Therefore the design principle of these metamaterials relies intimately on creation of appropriate local resonances and their interplay with background waves. In this review, we show that the coupling between rotation and bulk deformations of two-dimensional chiral solids is able to provide a unique resonant mechanism in designing elastic metamaterials, and the recent advances in this area will be reviewed. We begin with a metamaterial with a single-negative parameter by integrating a chiral lattice with resonating inclusions, and demonstrate that this metamaterial not only is suitable for broadband vibration isolation but also provides a mixed-type resonance due to microstructure chirality. This mixed-type resonance is further explored to realize elastic metamaterial with double-negative parameters, which can refract elastic wave with a negative refraction angle. Finally, we present also recent development on micropolar constitutive models, which are potentially suitable for modeling chiral elastic metamaterials.

Keywords: elastic metamaterials, chiral solids, negative refraction, micropolar theory

Highlights

- Recent advances in design of elastic metamaterials making use of chirality are reviewed.

- The bulk-rotational coupled mechanism of two dimensional chiral solids provides a distinct principle realizing doubly negative elastic metamaterial which is feasible to be engineered.

- $\quad$ The chiral lattice integrated with resonators is also a highly designable vibration isolating candidate.

- Chirality demands more sophisticated continuum theory, e.g. micropolar theory, in order to fully characterize its wave behavior.
\end{abstract}

\section{O INTRODUCTION}

Metamaterials are manmade composites with exotic properties not found in nature through deliberate microstructure design. There have been intensive research activities on these materials during the past decade. The breakthrough on metamaterials should be attributed to Pendry and his co-workers, they designed respectively electromagnetic (EM) composites with metallic wires and split-ring resonators [1] and [2] which can exhibit effectively negative permittivity and permeability in certain frequency ranges. In addition, EM metamaterials with simultaneously negative material parameters are termed as left handed materials (LHMs) and they are featured by the property that a wave traveling through such material will display anti-parallel phase and groupvelocity directions. For LHM, a number of exotic phenomena such as negative refraction, reversed Doppler-effect and Cherenkov radiation [3] and [4] are demonstrated theoretically or experimentally. Since metamaterials significantly enlarge material space available in designing wave-control devices, extensive investigations were carried out in the corresponding resonant mechanism, dynamic homogenization theory and microstructural design. The rapid advance of metamaterials was also boosted by the advent of transformation theory [5] and [6], which maps a deformed space into stringent distribution of material property and provides a superior methodology in designing wave devices. This and the metamaterial technique lead to a number of potential applications, perhaps the most creative examples are the invisible cloaking and the super lensing [7] and [8]. Similar ideas are naturally extended to conceive mechanical (acoustic/elastic) metamaterials.

The first elastic metamaterial (EMM) was proposed by embedding periodically silicon rubber coated lead spheres into a polymer matrix [9]. The resulting sample of the crystal can prohibit sound transmission in very low frequency range for which the corresponding wave length is over the crystal constant by two order of magnitudes. The underlying mechanism is obviously not due to the Bragg's scattering of traditional phononic crystals, but should be attributed to effective negative mass density (NMD) [10]. To understand the corresponding mechanism, Milton and Willis [11] constructed an illustrative mass-spring model, and showed that the NMD is due to the out-of-phase motion between the observable and hidden parts of microstructure near the resonating frequency. Yao et al. [12] experimentally examined the model and confirmed NMD at the bandgap region of a finite periodic system composed of mass-spring 
units. A Drude-model of NMD, for which the effective density is all negative below a cut-off frequency, is also found by letting the hidden mass infinitely large (unable to move). Based on this model, Yao et al. [13] designed a subwavelength structure with excellent sound insulating efficiency in very low frequency range. Yang et al. [13] proposed another scheme of NMD by tuning the out-of-plane resonant pattern of a strained membrane attached with small weights, and the NMD is defined by the ratio between the averaged acceleration and restoring force normal to the membrane. Membrane-type EMMs show a great advantage in sound insulation and absorption with lightweight structures [14] and [15]. Milton and Willis [11] also proved that dynamic effective density can also be a tensor by introducing different resonances along perpendicular directions. Huang and Sun [16] modeled the lattice system as an equivalent $2 \mathrm{D}$ elastic solid with its effective mass density characterized as a second order tensor. A continuum EMM with anisotropic effective dynamic mass density tensor was also proposed by placing lead cylinders coated with elliptical shaped rubbers in a matrix material [17] and [18]. Anisotropic mass density can also be achieved in non-resonant acoustic metamaterials containing fluid components [19] and [20]. On the other hand, Fang et al. [21] designed an acoustic metamaterial by using a one-dimensional array of subwavelength Helmholtz resonators, and found the abnormal transmission behavior in certain frequency range which can be explained by the effective negative modulus of the acoustic fluid.

However, synthesizing the known mechanisms achieving exotic elastic properties to build a bulk EMM with combined NMD, negative bulk modulus (NBM), negative shear modulus (NSM) or desired anisotropy is more complex compared to the EM media, this is mainly due to the inherent coupling between longitudinal and shear wave modes and wave mode conversion. In order to understand the internal mechanism of bulk EMMs, a systematic approach is to study the wave behavior of matrix-inclusion systems with coated sphere or cylinders, for which the analytical scattering solution is available [22] and [23]. It is now clear that the NBN, NMD and NSM are essentially related to the excited local resonant modes of monopolar, dipolar and quadrupolar types, respectively. In this regard, realization of acoustic metamaterial with doubly or triply negative parameters needs the simultaneous activation of more than one types of resonance within a certain frequency band, this either requires mixed fluid and solid components or complicated microstructure. Ding et al.
[24] proposed a double-negative acoustic metamaterial by combining an array of alternating bubble-contained water spheres and rubber-coated gold spheres in an epoxy matrix, each composite spheres are carefully designed to have an overlapped frequency of monopole resonance for bubble-contained water spheres and of dipole resonance for rubber-coated gold spheres. Other similar design schemes were proposed [25] and [26] as well. Wu et al. [27] proposed a type EMM by placing rubber coated water beads into a foam matrix, which can possess simultaneous NMD and NSM. Lai et al. [28] designed a hybrid solid made of four types of solid materials, which can selectively trigger the aforementioned resonances with wave direction. However, due to the fluid-solid combination and complex integrity, these EMM designs are theoretically successful but practically remain difficult to be realized for experimental demonstration. By using two-dimensional (2D) network of Helmholtz resonators, Zhang et al. experimentally demonstrated that the acoustic fluid media in the network can possess necessary double negativity or anisotropy for subwavelength imaging [29] or invisible cloaking [30]. Many other EMM based devices have been suggested for promising applications with elastic wave manipulations such as: elastic metamaterials waveguides, elastic wave super lensing, wave cloaking and vibration insulating [31] to [34].

Recently, it is evidenced that a new design principle different from the mentioned resonant scheme can be pursued if microstructure chirality is introduced. In this review, we will summarize the related works. Chirality was first termed by Lord Kelvin [35]: I call any geometrical figure, or group of points, 'chiral', and say that it has chirality if its image in a plane mirror, ideally realized, cannot be brought to coincide with itself. Lots of examples of chirality can be found in nature or manmade objects, such as twisted ropes and chiral nanotubes for threedimensional (3D) case, triskelion patterns for 2D case, respectively. It should be mentioned that the chirality have been employed earlier in realizing metamaterials to generate negative refraction for EM [36] and for acoustic cases as well [37], which results from the breaking of degeneracy between two circular polarized waves. The mechanism explored in this paper for EMM is however different. As will be demonstrated in the following, an outstanding feature of $2 \mathrm{D}$ chiral solids is the coupling between bulk deformation and local rotation. The feature enables the excitation of mixed translational and rotational resonances in the specially designed microstructure, 
and can be employed to realize double-negative EMMs in a simple and practical manner.

The paper is organized in five sections including this introduction. In Section 2, single-negative metamaterials are presented by integrating 2D chiral lattice with local resonators, their applications in broadband vibration isolating are also studied; in Section 3, the mechanism of mixed resonance and simultaneous NMD and NBM is first clarified by discrete models, and the design of realizable continuum versions of double-negative EMMs is given; in Section 4, recent developments on micropolar constitutive model which is potentially more suitable for characterizing chiral elastic materials and chiral EMMs are reported. Finally conclusions are provided in Section 5.

\section{SINGLE-NEGATIVE CHIRAL EMM}

2D periodic chiral lattice is not only a lightweight material with unique property for static load bearing, but also a highly designable phononic crystal with flexibly topological adaptation [38] and [39]. In particular, tri-chiral lattices, originally proposed by Prall and Lakes [40] to achieve negative Poisson's ratio, are received intensive investigations as ideal candidates for so-called auxetic materials. The geometry of tri-chiral lattice is depicted in Fig. 1 a), where a hexagonal unit cell is highlighted. The lattice is defined by circles of equal radius $r$ linked by straight ligaments of equal length $L$. The ligaments are required to be tangential to the circles and the angle between adjacent ligaments is equally 60 degrees. The distance between circle centers is denoted as $R$, while the angle between the line connecting the circle centers and the ligaments is defined as $\beta$. The thickness of the lattice ligaments is denoted as $t_{\mathrm{b}}$. The lattice vectors $\mathbf{e}_{i}$ can be written in the orthogonal Cartesian vector basis $\left(\mathbf{i}_{1}, \mathbf{i}_{2}\right)$ as

$$
\mathbf{e}_{1}=\left(\sqrt{3} \mathbf{i}_{1}+\mathbf{i}_{2}\right) R / 2, \quad \mathbf{e}_{2}=\left(-\sqrt{3} \mathbf{i}_{1}+\mathbf{i}_{2}\right) R / 2 .
$$

The ratio $\cos \beta=L / R$ is denoted as the topology parameter. By tuning continuously this parameter, a variety of distinct configurations, form traditional triangular lattice to packed circles, can be obtained as shown in Fig. $1 b$.

In order to achieve low-frequency wave attenuation, Liu et al. [41] proposed an EMM model by integrating a tri-chiral lattice with softly coated inclusions, which function as NMD resonators, in the hollow circles. The unit cell of the EMM lattice is shown in Fig. 1c, and the radius of the core cylinder is identified as $r_{\mathrm{c}}$. The first Brillouin zone, the reciprocal lattice vectors $\left(\mathbf{b}_{1}, \mathbf{b}_{2}\right)$ and the irreducible Brillouin zone (IBZ) of the lattice are shown in Fig. 1d. To study the working mechanism of the proposed singlenegative chiral EMM, band structures are calculated by using finite element based Bloch wave analysis. The configuration of the analyzed latticed composite, including geometry and material parameters, are detailed in Table 1. In the figure, results are presented in the normalized frequency $\Omega=\omega / \omega_{0}$ with $\omega_{0}$ being the first order flexural frequency of a simply supported ligament of length $L$.
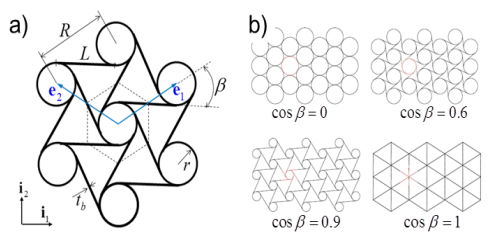

c)

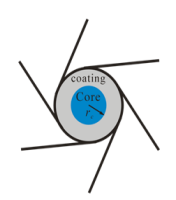

d)

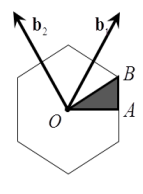

Fig. 1. a) Geometry of the tri-chiral lattice; b) different lattice configurations induced by variation of the topology parameter; c) unit cell of the EMM lattice; d) reciprocal lattice vectors and Brillouin zone; taken from Liu et al. [41]

Table 1. Geometric and material parameters of the single negative chiral EMM lattice

\begin{tabular}{|c|c|c|}
\hline \multirow{7}{*}{$\begin{array}{l}\text { Lattice } \\
\text { parameters }\end{array}$} & topology parameter & $L / R=0.9$ \\
\hline & ligament length & $=26.4 \mathrm{~mm}$ \\
\hline & node radius & $=6.4 \mathrm{~mm}$ \\
\hline & ligament wall thickness & $t_{\mathrm{b}}=0.5 \mathrm{~mm}$ \\
\hline & Young's modulus & $E_{1}=71 \mathrm{GPa}$ \\
\hline & Poisson's ratio & $v_{1}=0.33$ \\
\hline & density & $\rho_{1}=2.7 \mathrm{~g} / \mathrm{m}^{3}$ \\
\hline \multirow{7}{*}{$\begin{array}{l}\text { Lattice } \\
\text { parameters }\end{array}$} & core-node radius ratio & $r_{\mathrm{c}} / r=0.5$ \\
\hline & core Young's modulus & $E_{\mathrm{c}}=17 \mathrm{GPa}$ \\
\hline & core Poisson's ratio & $v_{\mathrm{c}}=0.33$ \\
\hline & core density & $\rho_{\mathrm{c}}=13 \mathrm{~g} / \mathrm{m}^{3}$ \\
\hline & coating Young's modulus & $E_{\mathrm{s}}=5 \mathrm{GPa}$ \\
\hline & coating Poisson's ratio & $v_{\mathrm{S}}=0.33$ \\
\hline & coating density & $\rho_{\mathrm{s}}=0.5 \mathrm{~g} / \mathrm{m}^{3}$ \\
\hline
\end{tabular}

The band structure of the chiral EMM is shown in Fig. 2a. For reference, the band diagram of a pure lattice structure without coated inclusions is also plotted in Fig. 2b. It is noticed that the band structures in the two lattice materials with and without 
resonators are almost unchanged at high frequency range $\Omega \in[2,6]$, while at low-frequency range, a considerably wide band gap $\Omega \in[0.81,1.43]$ is found for the former. Dispersion curves of the first two lowest modes, which correspond to P (longitudinal) and $\mathrm{S}$ (transverse) modes of the pure lattice, are also plotted in Fig. 2a by dashed lines. It is seen that the lowest two branches are split into five branches due to the presence of local resonators.
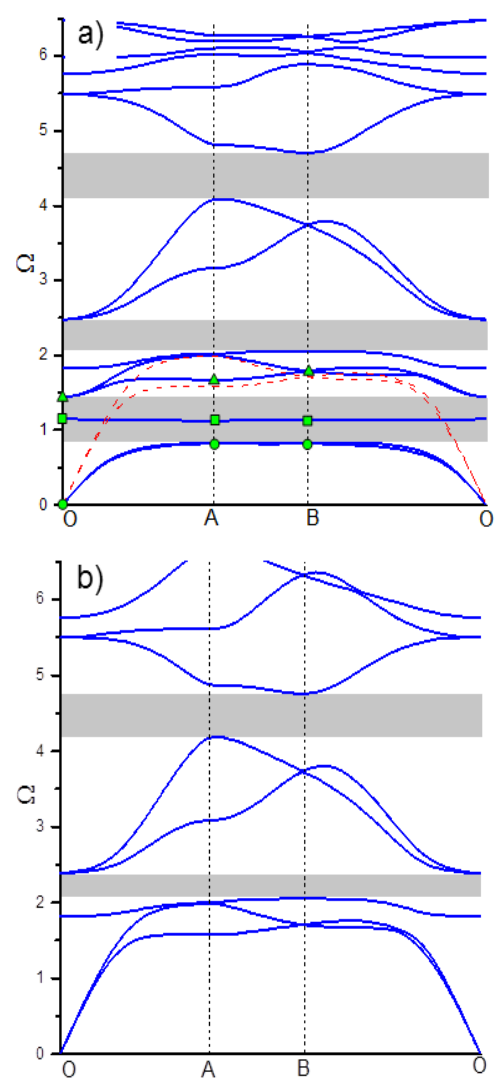

Fig. 2. Band diagrams of the lattice a) with and b) without coated inclusions; taken from Liu et al. [41] (For interpretation of the colors in figures, the reader is referred to the web version of this article)

Wave modes are selectively presented in Fig. 3 to further understand the formation of the low-frequency band gap. In the figure, mode shapes of the first, third and fourth branches located at the high-symmetry points $(\mathrm{O}, \mathrm{A}, \mathrm{B})$ of the IBZ, which are highlighted in Fig. 2a by dots, are displayed. Un-deformed geometry (red line) is imposed as reference. The first and fourth branches form the boundaries of the first bandgap. The points $\mathrm{A}$ and $\mathrm{B}$ in the first branch and the point $\mathrm{O}$ in the fourth branch are on the edge of the band gap, for which it can be seen that the mechanism of the wave attenuation is the translational resonance of the core in the soft coating layer and the deformation of the lattice is small. In this case, most part of the wave energy is trapped in the coated inclusion due to its local resonance and wave propagation is not allowed. For the locus away from the edge of the band gap, point $\mathrm{O}$ in the first branch corresponds to the rigid mode of the structure, while the modes of point A and B in the fourth branch show the propagating wave takes place through the bending of the lattice ligaments.

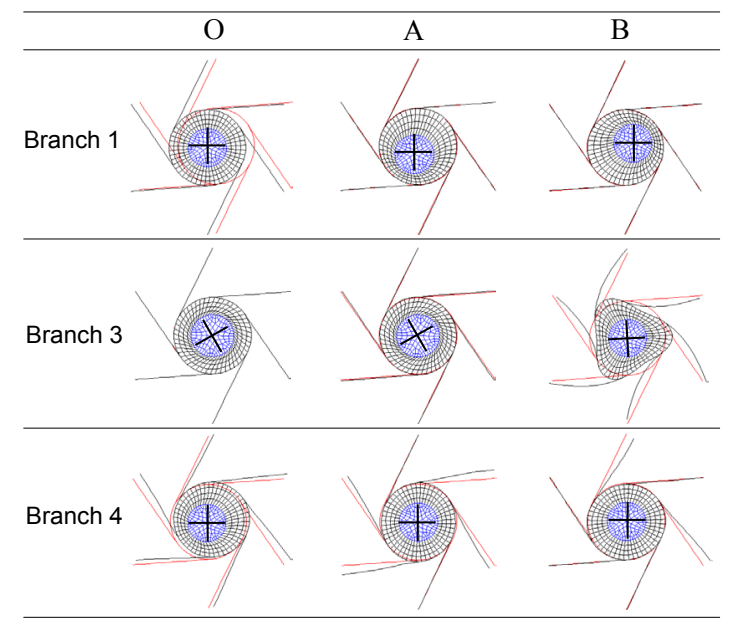

Fig. 3. Mode shapes of three typical branches at high-symmetry points of the Brillouin zone; taken from Liu et al. [41]

The third branch in Fig. 2a is remarkable. It is found that the slope of this very narrow passing band $\Omega \in[1.14,1.15]$ along the OA path is negative, which implies that the anti-parallel directions of group and phase velocity. Moreover, this negative band takes place in the low frequency range where the effective mass density of the EMM is negative, thus it is more appropriate to reason that in this band the effective modulus turns negative as well. The wave modes in Fig. 3 indicate that the formation of this branch is closely associated to the rotational motion of the core cylinder. It is also worthwhile to point out that as long as the core possessed a rotational inertia, the third rotation related branch always exists in the band diagram. However, if the chirality is not present, the dispersion curve of this branch will be strictly horizontal, which implies that the group velocity is zero and the rotational mode actually cannot be excited. Analysis of rotation related band is placed in Section 2.1.

In order to test vibration alleviation effect of the previous chiral EMM at low frequency, Zhu et al. [42] experimentally investigated the frequency response of a finite sized beam structure. The sandwiched beam 
consists of a periodic tri-chiral lattice sandwiched into a rectangular frame $(470 \mathrm{~mm}$ in length, 91 $\mathrm{mm}$ in height, $10 \mathrm{~mm}$ in width and $0.5 \mathrm{~mm}$ in wall thickness), which is fabricated from an aluminum ( $\mathrm{Al}$ ) plate through a water jet cutter. Local resonators made of rubber (Polyteks Poly PT Flex 20 RTV Liquid Rubber) coated metal cylinders are inserted in lattice beam. Steel and tungsten cylinders with the same geometry are used as inclusion cores for the purpose of generating different resonant frequencies. The EMM parameters are specified by Table 2. A specimen containing two sections of different resonators, which is fabricated to test the broadband suppression of vibration, is shown in Fig. 4a. In the experiment, the beam is fixed on one end and excited by a shaker close to the fixed end. White noise excitation signal with bandwidth from 0 to $1000 \mathrm{~Hz}$ is generated by the shaker, and the response of the EMM beam is received by an accelerometer attached to the other end. Band structure analysis is conducted as well for comparison.

Table 2. Parameters of fabricated chiral EMM lattice with metamaterial resonators

\begin{tabular}{|c|c|c|c|}
\hline \multirow{7}{*}{$\begin{array}{l}\text { Lattice } \\
\text { parameters }\end{array}$} & \multirow{2}{*}{$\begin{array}{l}\text { topology parameter } \\
\text { ligament length }\end{array}$} & \multicolumn{2}{|c|}{$L / R=0.82$} \\
\hline & & $L$ & $=24.6 \mathrm{~mm}$ \\
\hline & node radius & $r$ & $=8.6 \mathrm{~mm}$ \\
\hline & ligament wall thickness & $t_{\mathrm{b}}$ & $=0.5 \mathrm{~mm}$ \\
\hline & Young's modulus & $E_{1}$ & $=71 \mathrm{GPa}$ \\
\hline & Poisson's ratio & $v_{1}$ & $=0.33$ \\
\hline & density & $\rho_{1}$ & $=2.7 \mathrm{~g} / \mathrm{m}^{3}$ \\
\hline \multirow{6}{*}{$\begin{array}{l}\text { Lattice } \\
\text { parameters }\end{array}$} & diameter of metal cylinder & & $6.35 \mathrm{~mm}$ \\
\hline & height of metal cylinder & & $25.4 \mathrm{~mm}$ \\
\hline & density of steel & & $7.85 \mathrm{~g} / \mathrm{m}^{3}$ \\
\hline & density of tungsten & & $15.63 \mathrm{~g} / \mathrm{m}^{3}$ \\
\hline & rubber Young's modulus & & $586 \mathrm{Ma}$ \\
\hline & loss tangent of rubber & & $<0.1$ \\
\hline
\end{tabular}

Fig. 4b shows the measured FRF of EMM beam with mixed resonators in solid line. For comparison, FRFs from EMM beam with single section of resonators (steel and tungsten cylinders) are also tested and plotted in dashed and dotted lines, respectively. It is found that the frequency region of attenuation of the EMM beam with mixed resonators falls in between $210 \mathrm{~Hz}$ and $700 \mathrm{~Hz}$, which is very close to the summation of those regions of the beams with individual resonator section. In the figure, yellow and gray shaded areas indicate the band gap prediction for infinite chiral EMMs with two types of the resonators, respectively. It is seen that the measured FRFs can be well predicted by either individual band gaps or their overlap correspondingly.
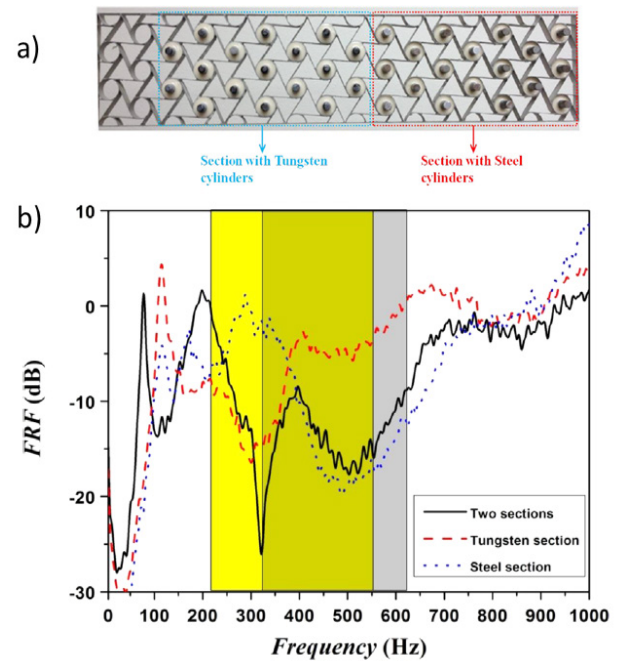

Fig. 4. a) Fabricated EMM beam structure with two sections of resonators; b) comparison of measured and predicted FRFs for the broadband EMM beam; taken from Zhu et al. [42]

\section{DOUBLE-NEGATIVE CHIRAL EMM}

It is seen that introducing chirality in EMMs can effectively activate the usually silent rotational mode of resonance in microstructure. This inspires a new mechanism to design EMMs with simultaneously negative NMD and NBM by making use of coupled translational and rotational resonances through appropriate microstructure design. The resulting double-negative EMM will contain only a single type of resonator, and will be considerably simpler than the traditional schemes.

\subsection{Discrete Models}

In order to illustrate how the negative effective modulus can be produced by rotational resonance, consider a 1D chiral mass-spring unit shown in Fig. 5. Three massless springs and a rigid disk with rotational inertia $I$ are pin-connected. The two springs with elastic constant $k_{2}$ are tangential to a rigid disk with an angle $\alpha$. During loading process, the pin-joints $A, C$ as well as the disk center are kept in the horizontal axis.

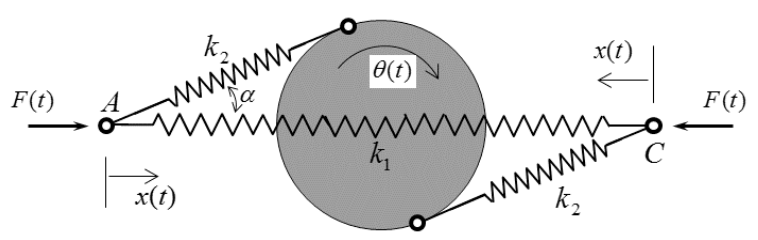

Fig. 5. $1 D$ discrete model demonstrating negative stiffness; taken from Liu et al. [43] 
The rotation of the disk is induced by the equally applied force $F$. The tension in the spring $k_{2}$ is given by:

$$
f_{2}=k_{2}(R \theta-x \cos \alpha)
$$

The rotation of the disk is governed by:

$$
2 f_{2} R=-I \frac{\partial^{2} \theta}{\partial t^{2}}
$$

with $R$ being the radius of the rigid disk. Assuming the system is time-harmonically loaded and all quantities have the form as $(F, x, \theta)=(\widehat{F}, \hat{x}, \hat{\theta}) e^{i \omega t}$, the rotation of the disk and the end displacement can be related as

$$
\hat{\theta}=\frac{2 R k_{2} \cos \alpha}{2 R^{2} k_{2}-\omega^{2} I} \hat{x}
$$

The balance of the force at the joint $A$ then gives:

$$
F=2 k_{1} x-f_{2} \cos \alpha .
$$

The dynamic effective stiffness of the system, defined by $k_{\text {eff }}=\widehat{F} /(2 \hat{x})$, reads:

$$
k_{e f f}=k_{1}+\frac{k_{2} \cos ^{2} \alpha}{2}\left(1-\frac{\omega_{0}^{2}}{\omega_{0}^{2}-\omega^{2}}\right),
$$

where $\omega_{0}=\sqrt{2 k_{2} R^{2} / I}$ represents the resonant frequency of the disk. It can be shown that the effective stiffness takes negative value in the following frequency range:

$$
\sqrt{2 k_{1} /\left(2 k_{1}+k_{2} \cos ^{2} \alpha\right)}<\frac{\omega}{\omega_{0}}<1 .
$$

The physics of the negative $k_{\text {eff }}$ can be explained as follows. Consider the system subjected to compressive deformation, that is, $\hat{x}$ is positive. Then Eqs. (4) and (7) give that:

$$
\frac{\left(2 k_{1}+k_{2} \cos ^{2} \alpha\right)}{k_{2} R \cos \alpha} \hat{x}<\hat{\theta}<+\infty,
$$

implying in-phase (clockwise) rotation of the disk. It turns out that though the spring force in $k_{1}$ is compressive $\left(\widehat{f}_{1}=-2 k_{1} \hat{x}\right)$, the spring force in $k_{2}$ can be tensile due to the intensive clockwise rotation of the disk. Specifically, by substituting Eq. (8) into Eq. (2) we obtain that $2 k_{1} \hat{x} / \cos \alpha<f_{2}<+\infty$, this is the reason why the external force feels tension while the system is shortened.

Next, the previous negative-stiffness unit is used to build a 1D discrete system with doublenegative effective properties, as shown in Fig. 6. The system consists of an infinite host chain of masses $m_{1}$ connected by springs $k_{1}$, and the resonators, which consist of mass $m_{2}$ and rotation inertia $I$ and two inclined springs $k_{2}$, are inserted in between the adjacent host masses. A dashed box in the figure highlights the $n^{\text {th }}$ unit cell in the system. Different from the previous negative stiffness model, here the disks will undergo not only rotation but also translation. Wang [44] considered a similar 1D discrete model in which two chiral resonators with different handedness are superimposed in a unit cell to eliminate the overall non-symmetric effect. The dynamic properties and wave behavior are thoroughly examined in their work. $\mathrm{Li}$ and Wang [45] later generalized the discrete model to $2 \mathrm{D}$ case.

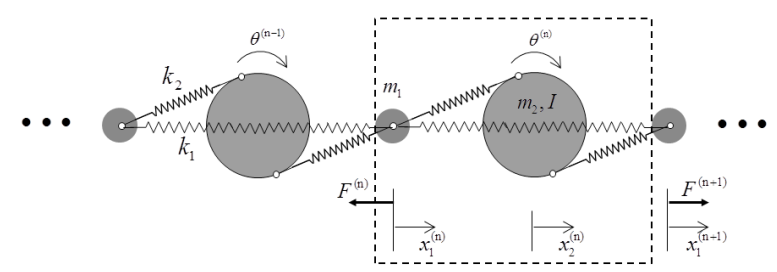

Fig. 6. $1 D$ discrete model demonstrating simultaneous NMD and stiffness

Assuming the harmonic response as before, the dynamic equation of the system can be characterized by the following equations:

$$
\begin{aligned}
-\omega^{2} m_{1} x_{1}^{(\mathrm{n})}= & k_{1}\left(x_{1}^{(\mathrm{n}+1)}+x_{1}^{(\mathrm{n}-1)}-2 x_{1}^{(\mathrm{n})}\right) \\
& +k_{2} R \cos \alpha\left(\theta^{(\mathrm{n})}-\theta^{(\mathrm{n}-1)}\right) \\
& +k_{2} \cos ^{2} \alpha\left(x_{2}^{(\mathrm{n})}+x_{2}^{(\mathrm{n}-1)}-2 x_{1}^{(\mathrm{n})}\right), \\
-\omega^{2} m_{2} x_{2}^{(\mathrm{n})}= & k_{2} \cos ^{2} \alpha\left(x_{1}^{(\mathrm{n}+1)}+x_{1}^{(\mathrm{n})}-2 x_{2}^{(\mathrm{n})}\right) \\
-\omega^{2} I \theta^{(\mathrm{n})}= & -R k_{2} \cos \alpha\left(x_{1}^{(\mathrm{n}+1)}-x_{1}^{(\mathrm{n})}\right)-2 R^{2} k_{2} \theta^{(\mathrm{n})} .
\end{aligned}
$$

From Eq. (9) the translation and rotation of the rigid disk are related with the displacement of the host masses as [46]:

$$
\begin{aligned}
& x_{2}^{(\mathrm{n})}=\frac{1}{1-\omega^{2} / \omega_{1}^{2}} \frac{x_{1}^{(\mathrm{n}+1)}+x_{1}^{(\mathrm{n})}}{2}, \\
& \theta^{(\mathrm{n})}=\frac{1}{1-\omega^{2} / \omega_{0}^{2}} \frac{x_{1}^{(\mathrm{n})}-x_{1}^{(\mathrm{n}+1)}}{2 R} \cos \alpha,
\end{aligned}
$$

where $\omega_{0}$ and $\omega_{1}$ are the natural frequencies of the rigid disk, given by:

$$
\omega_{0}^{2}=\frac{2 k_{2} R^{2}}{I}, \quad \omega_{1}^{2}=\frac{2 k_{2} \cos ^{2} \alpha}{m_{2}} .
$$


By analyzing the dynamic motion of the host masses in conjunction of the boundary forces $F^{(\mathrm{n}+1)}$ and $F^{(\mathrm{n})}$ acted on unit cell, the following relations can be obtained:

$$
\begin{aligned}
\frac{1}{2}\left(F^{(\mathrm{n}+1)}+F^{(\mathrm{n})}\right) & =\left(k_{1}-\frac{m_{1}}{4} \omega^{2}+\frac{k_{2} \cos ^{2} \alpha}{2} \frac{1}{1-\omega_{0}^{2} / \omega^{2}}\right) \\
& \times\left(x_{1}^{(\mathrm{n}+1)}-x_{1}^{(\mathrm{n})}\right), \\
F^{(\mathrm{n}+1)}-F^{(\mathrm{n})} & =-\omega^{2}\left(m_{1}+\frac{m_{2}}{1-\omega^{2} / \omega_{1}^{2}}\right) \frac{x_{1}^{(\mathrm{n}+1)}+x_{1}^{(\mathrm{n})}}{2} .
\end{aligned}
$$

Bearing in mind that the average tension is $\left(F^{(\mathrm{n}+1)}+F^{(\mathrm{n})}\right) / 2$, elongation is $x_{1}^{(\mathrm{n}+1)}-x_{1}^{(\mathrm{n})}$, total force is $\left(F^{(\mathrm{n}+1)}-F^{(\mathrm{n})}\right)$, and the average observable acceleration is $-\omega^{2}\left(x_{1}^{(\mathrm{n}+1)}+x_{1}^{(\mathrm{n})}\right) / 2$, the effective stiffness and mass of the system are obviously;

$$
\begin{gathered}
k_{e f f}=k_{1}-\frac{m_{1}}{4} \omega^{2}+\frac{k_{2} \cos ^{2} \alpha}{2} \frac{1}{1-\omega_{0}^{2} / \omega^{2}}, \\
m_{e f f}=m_{1}+\frac{m_{2}}{1-\omega^{2} / \omega_{1}^{2}} .
\end{gathered}
$$

The harmonic wave solution of the 1D system can be expressed as:

$$
\left(x_{1}^{(\mathrm{n}+j)}, x_{2}^{(\mathrm{n}+j)}, \theta^{(n+j)}\right)=\left(\hat{x}_{1}, \hat{x}_{2}, \hat{\theta}\right) e^{i(q x+j q L-\omega t)},
$$

where $q$ is the Bloch wave number, $L$ is the length of the unit cell and $\left(\hat{x}_{1}, \hat{x}_{2}, \hat{\theta}\right)$ are complex amplitudes. By substituting Eq. (15) into Eq. (9) and solving the eigenvalue problem of the coefficients, the dispersion relation can be expressed as:

$$
\begin{array}{r}
\omega^{2}\left(m_{1}+\frac{m_{2}}{1-\omega^{2} / \omega_{1}^{2}}\right)=\left[k_{1}+\frac{k_{2} \cos ^{2} \alpha}{2}\right. \\
\left.\left(\frac{\omega_{1}^{2}}{\omega_{1}^{2}-\omega^{2}}-\frac{\omega_{0}^{2}}{\omega_{0}^{2}-\omega^{2}}\right)\right] \sin ^{2} \frac{q L}{2},
\end{array}
$$

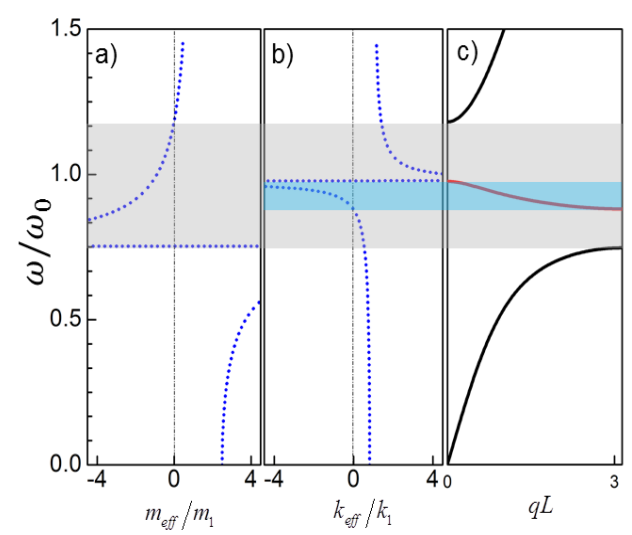

Fig. 7. a) Effective mass, b) stiffness and c) dispersion curves of the doubly negative $1 D$ chiral discrete model
Thus, the dispersion curves are plotted according to Eq. (16) in Fig. 7c with the parameters as following: $L=0.1, R=0.08, \alpha=\pi / 6, m_{1}=0.2, m_{2}=0.3, I=0.0015$, $k_{1}=0.2$, and $k_{2}=0.1$. It can be found in Fig. $7 \mathrm{c}$ that a pass band with negative slope (red solid line) within frequency range $(0.88$ to 1.00$)$ is generated inside the bandgap region and separates the bandgap region into two small gaps at (0.75 to 0.88$)$ and (1.00 to 1.18). Fig. 7a shows the normalized effective mass $\left(m_{\text {eff }} / m_{1}\right)$ as function of frequency predicted by Eq. (14), the negative effective mass is found at frequency range ( 0.85 to 1.18$)$, highlighted with grey color in the figure. Fig. $7 \mathrm{~b}$ shows the normalized effective stiffness calculated by Eq. (13), the negative stiffness is found at frequency range ( 0.90 to 1.00$)$. It is noticed that the frequency range where negative effective mass and stiffness occurs simultaneously is almost overlapped with that of the pass band with negative slope. On the other hand, the separated two small gaps agree well with the regions where only negative mass exists.

\subsection{Three-Phase Chiral EMM with Double-Negative Property}

Enlightened by the discrete model of Fig. 6, Liu et al. [43] proposed a 2D continuum version of EMM with simultaneously NMD and NBM. The analogy between 1D discrete model and continuum EMM is illustrated in Fig. 8a. The host lattice of discrete model is replaced by continuum matrix material with cavities where the resonators is inserted, while the chiral coating mimics the inclined springs of resonators. To make the EMM macroscopically isotropic, the resonators are arranged in a periodic triangular pattern with lattice constant $a$. The unit cell of the metamaterial is depicted in Fig. $8 \mathrm{~b}$. A number of $\left(n_{\mathrm{s}}\right)$ slots with width $t_{\mathrm{s}}$ are cut out from the coating material. The slots are equi-spaced in azimuth and oriented at an angle $\theta_{\mathrm{s}}$ with respect to the radial direction. The metamaterial lacks any planes of mirror symmetry hence it is said to be

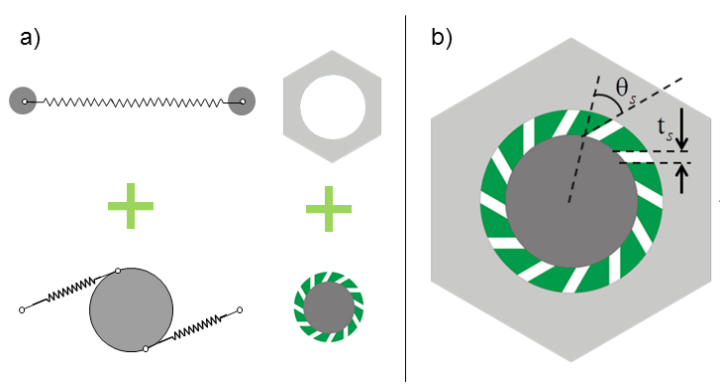

Fig. 8. a) Analogy between the $1 D$ discrete model and $2 D$ continuum EMM; b) unit cell configuration of chiral EMM; taken from Liu et al. [43] 
chiral. The geometrical parameters mentioned above as well as the constituent materials were carefully designed to ensure the translational and rotational resonances occur at overlapped frequency range, and the overlapped frequency was further optimized to be as large as possible.

In order to evaluate dynamic effective properties of the EMM with such a complicated microstructure, analytical method is not available. Instead, a numerical-based effective medium method based on the micromechanics approach is adopted. Under longwavelength approximation, the macroscopic stress, strain, resultant force and acceleration of the unit cell can be determined by averaging local quantities on the unit cell's external boundary as [46]:

$$
\begin{array}{ll}
\Sigma_{\alpha \beta}=\frac{1}{V} \int_{\partial V} \sigma_{\alpha \gamma} x_{\beta} d s_{\gamma}, & E_{\alpha \beta}=\frac{1}{2 V} \int_{\partial V}\left(u_{\alpha} d s_{\beta}+u_{\beta} d s_{\alpha}\right), \\
F_{\alpha}=\frac{1}{V} \int_{\partial V} \sigma_{\alpha \beta} d s_{\beta}, & \ddot{U}_{\alpha}=\frac{1}{S} \int_{\partial V} \ddot{u}_{\alpha} d s,
\end{array}
$$

where the Einstein's summation rule on the repeated subscripts is assumed and Greek subscripts range from 1 to $2 ; \sigma_{\alpha \beta}, u_{\alpha}$ and $\ddot{u}_{\alpha}$ are the local stress, displacement and acceleration fields, respectively; $\mathrm{d} s_{\alpha}=n_{\alpha} \mathrm{d} s$ with $n_{\alpha}$ and $\mathrm{d} s$ being the boundary unit normal and line element of the boundary, respectively; $x_{\alpha}$ and $V$ denote the position vector and unit cell's volume, respectively. Considering the macroscopic isotropy, the effective bulk, shear modulus and momentum mass density of the EMM can be defined as:

$$
\begin{aligned}
& K_{\text {eff }}=\frac{1}{2} \Sigma_{\alpha \alpha} / E_{\alpha \alpha}, \quad \mu_{e f f}=\frac{1}{2} \Sigma_{\alpha \beta}^{\prime} / E_{\alpha \beta}^{\prime}, \\
& \rho_{\text {eff }}=F_{\alpha} / \ddot{U}_{\alpha},
\end{aligned}
$$

where $\Sigma_{\alpha \beta}^{\prime}$ and $E_{\alpha \beta}^{\prime}$ denote the deviatoric parts of the macroscopic stress and strain, respectively. To evaluate the dynamic properties, the unit cell is time-harmonically loaded on the boundary by the prescribed displacement:

$$
u_{\alpha}(\mathbf{x}, t)=\hat{u}_{\alpha}(\mathbf{x}) e^{i \omega t}, \quad \hat{u}_{\alpha}(\mathbf{x})=\hat{u}_{\alpha}^{0}+\hat{E}_{\alpha \beta} x_{\beta},
$$

where the boundary displacement amplitude $\hat{u}_{\alpha}$ is required to be compatible with a known macro-strain amplitude $\widehat{E}_{\alpha \beta}$. The dynamics of the unit cell is then solved by harmonic finite element analysis with frequency being swept over the interested range, from which the effective properties are determined by Eq. (18).

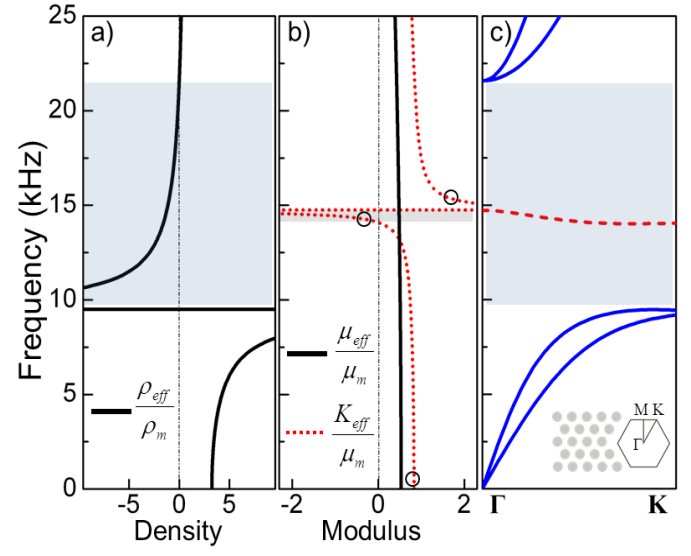

Fig. 9. a) Effective density, b) effective moduli and c) band structure of the three-material chiral elastic metamaterial; taken from Liu et al. [43]

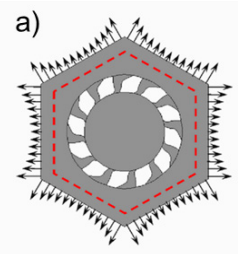

b)

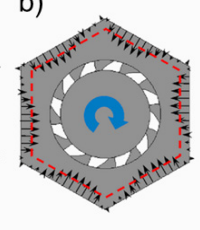

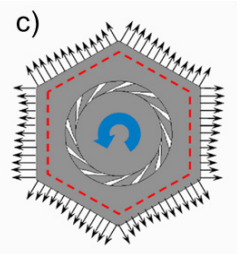

Fig. 10. Deformation and rotational resonance modes corresponding to three typical values of the effective dynamic bulk modulus: a) quasi-static value, b) negative value and c) positive peak; taken from Liu et al. [43]

An example of the final EMM design is specified with the following parameters: epoxy resin (density $\rho_{m}=1110 \mathrm{~kg} / \mathrm{m}^{3}$, bulk modulus $K_{m}=3.14 \mathrm{GPa}$, shear modulus $\mu_{m}=0.89 \mathrm{GPa}$ ), low-density Polyethylene $\left(\rho_{c}=920 \mathrm{~kg} / \mathrm{m}^{3}, K_{c}=0.57 \mathrm{GPa}, \mu_{c}=0.13 \mathrm{GPa}\right)$ and lead $\left(\rho_{i}=11600 \mathrm{~kg} / \mathrm{m}^{3}, K_{i}=52.6 \mathrm{GPa}, \mu_{i}=14.9 \mathrm{GPa}\right)$ are chosen as matrix, coating and core materials, respectively; the core is $5.6 \mathrm{~mm}$ in diameter and the coating thickness is $0.7 \mathrm{~mm}$; the triangular lattice constant is $a=10.75 \mathrm{~mm}$; the slot parameters are $n_{s}=12, t_{s}=0.4 \mathrm{~mm}$ and $\theta_{\mathrm{s}}=56^{\circ}$, respectively. Fig. $9 \mathrm{a}$ shows normalized effective mass density $\rho_{\text {eff }} / \rho_{m}$ of the EMM as function of wave frequency. It is seen that the negative density appears in range of $9.51 \mathrm{kHz}$ to $21.54 \mathrm{kHz}$. Fig. 9b shows the normalized effective bulk modulus $K_{\text {eff }} / \mu_{m}$ and shear modulus $\mu_{\text {eff }} / \mu_{m}$ as a function of frequency. It is of interest to note that $K_{\text {eff }}$ becomes negative in frequency range of $14.08 \mathrm{kHz}$ to $14.72 \mathrm{kHz}$ while $\mu_{\text {eff }}$ is always positive. The wave dispersion relation along $\Gamma \mathrm{K}$ direction is shown in Fig. 9c. The lattice array and the IBZ are also plotted as the inset of Fig. 9c. A stop band is observed for both longitudinal and transverse waves in the frequency range of $9.44 \mathrm{kHz}$ to $21.58 \mathrm{kHz}$, which matches the 
frequency range of negative effective density quite well. In addition, a new pass band with negative slope appears in the frequency range of $14.05 \mathrm{kHz}$ to $14.73 \mathrm{kHz}$, which again matches the overlapped frequency range of the negative effective density and longitudinal modulus $\left(K_{\text {eff }}+\mu_{\text {eff }}\right)$ well.

The origin of the NBM of the proposed EMM can be understood by examining the deformation and traction of a unit cell corresponding to three typical values of $K_{\text {eff }}$ as shown in Fig. 10. The undeformed states are identified by dashed lines. In Fig. $10 \mathrm{a}(0 \mathrm{kHz})$, a quasi-static $K_{\text {eff }}$ is obtained since the frequency is far from the core rotational resonance frequency. When the frequency approaches the rotational resonance frequency from below (14.5 $\mathrm{kHz}$ ), a very large clockwise (in-phase) rotation of the core is generated in conjunction with expansion of the unit cell. Such a rotation produces a compressive state in the matrix and compression is also detected on the external boundary of the unit cell. Conversely in Fig. $10 \mathrm{c}$, when the frequency approaches the rotational resonance frequency from above $(15.2 \mathrm{kHz})$, the anticlockwise rotation of the core enhances the tensile state in the matrix and, consequently, a positive peak of $K_{\text {eff }}$ occurs.

\subsection{Single-Phase Chiral EMM with Double-Negative Property}

The chirality and coupled translational and rotational resonances can be employed to design doublenegative EMMs through a single type of unit cell. This could simplify significantly the design of EMMs, however the microstructural pattern given in the previous section is still too complicated for experimental validation. To this end, Zhu et al. [47] proposed an innovative chiral EMM design made of only one single solid material, which is easy to be fabricated and tested in plate-based techniques. The unit cell of the proposed EMM is shown in Fig. $11 \mathrm{a}$, which is composed of one matrix material and slotted voids. The chirality of the unit cell is formed by properly slot-cutting in a hexagonal area and leaving three inclined ribs which connect the center piece (functioned as the mass) and the frame. The lattice constant is $a$, and the widths of the slot, ribs and frames are denoted by $s, r$ and $t_{f}$, respectively. In the design, the three ribs are very critical since they function as the soft chiral coating introduced in the previous example, and they will support not only the translation resonance but also the rotation resonance of the center piece. Therefore, by carefully choosing the base material and optimizing the geometrical parameters, it is possible to simultaneously achieve translation and rotation resonances and in turn the double negativity at a desired frequency range.

A practical example of the proposed singlephase EMM is specified as following: stainless steel with density $7850 \mathrm{~kg} / \mathrm{m}^{3}$, Young's modulus $200 \mathrm{GPa}$, Poisson's ratio 0.3 is chosen as the base material; the size of unit cell and other geometric parameter are $a=12 \mathrm{~mm}, s=0.5 \mathrm{~mm}, r=0.4 \mathrm{~mm}$ and $t_{f}=1.2$ $\mathrm{mm}$, respectively. The effective bulk modulus and the effective mass density of the chiral EMM are evaluated by the method explained in the previous section, and plotted as a function of frequency in Figs. $11 \mathrm{c}$ and d, respectively. The effective mass density $\rho_{\text {eff }}$ and effective bulk modulus $K_{\text {eff }}$ are normalized with the density and Young's modulus of the base material, respectively. The dispersion curves along $Г \mathrm{M}$ direction are also graphed in Fig. 11b, in which the lattice array and its IBZ are also inserted as the inset of Fig. 11b. It is found that the bandgap frequency range (grey area in $37.2 \mathrm{kHz}$ to $53.6 \mathrm{kHz}$ ) predicted from the dispersion relation is almost the same as the frequency region (pink area in $37.4 \mathrm{kHz}$ to $54.1 \mathrm{kHz}$ ) of the NMD. In Fig. $11 \mathrm{c}, K_{\text {eff }}$ turns negative in a frequency range of $42.9 \mathrm{kHz}$ to $45.2 \mathrm{kHz}$ (blue strip). Eventually, both the effective longitudinal modulus $E_{\text {eff }}=K_{\text {eff }}+\mu_{\text {eff }}$ and $\rho_{\text {eff }}$ become negative in the regime of $43.6 \mathrm{kHz}$ to $45.2 \mathrm{kHz}$, which implies a pass band with negative

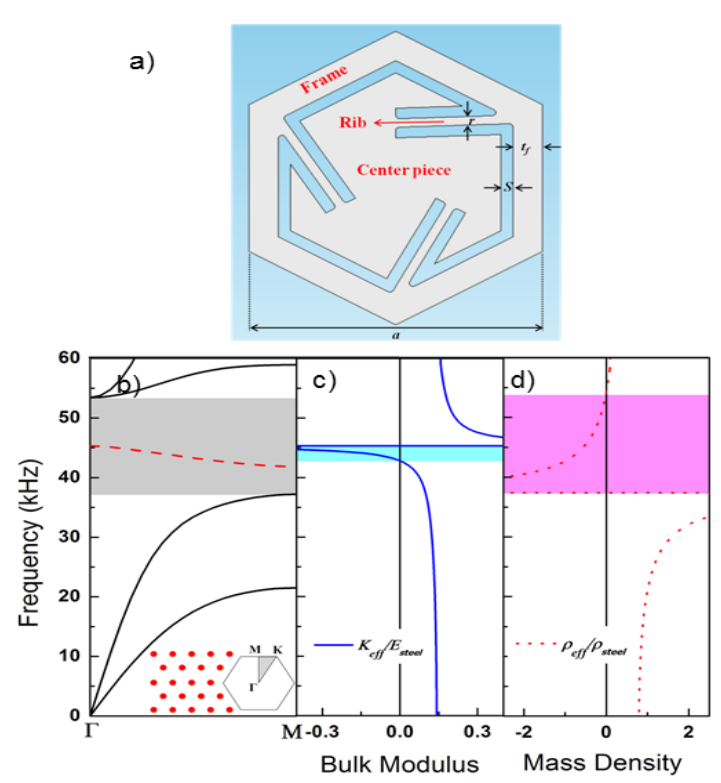

Fig. 11. a) The unit cell of the single-phase chiral EMM; b) band structure; c) effective bulk modulus and d) effective density of the proposed chiral elastic metamaterial; taken from Zhu et al. [47] 
slope for longitudinal wave in this frequency range. The longitudinal wavelength belonging to this band is from $112 \mathrm{~mm}$ to $121 \mathrm{~mm}$, and it is much larger than the unit cell size $a=12 \mathrm{~mm}$.

A prominent feature of double-negative metamaterial is the negative refraction phenomenon when a propagating wave impinges the interface between the metamaterial and a normal media, originated from the antiparallel direction of energy flow and phase velocity as well as conservation of transverse component of the wave vector. The double negativity of the proposed chiral EMM was numerically and experimentally justified by the negative refraction between an EMM and a normal elastic material. In the simulation, a 30-degree wedged sample composed of 512 EMM unit cells is constructed in a surrounding normal elastic material which is the same as the base material of the EMM, see Fig. 12b. A longitudinal wave beam is generated from the left side edge of the EMM wedge by applying a harmonic displacement excitation. The wave beam propagates inside the metamaterial, and eventually refracts at the inclined edge. The simulation is performed in COMSOL Multi Physics.
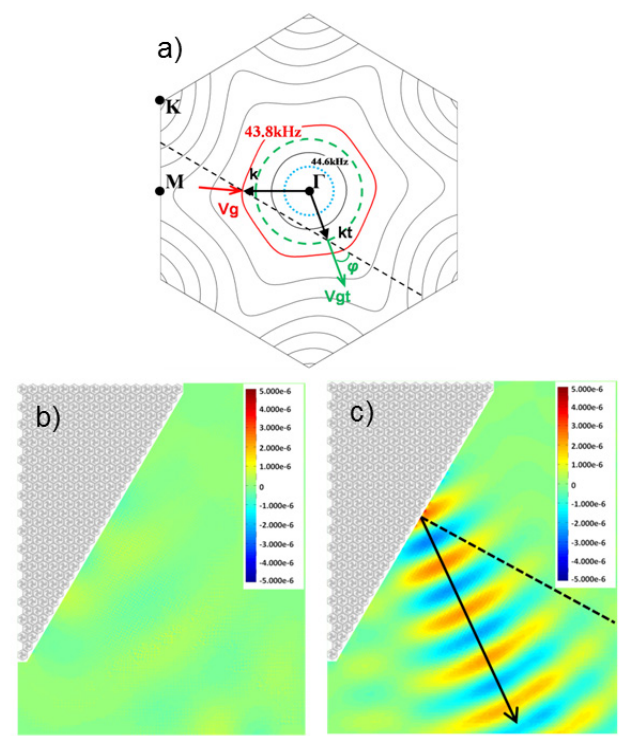

Fig. 12. a) The EFCs of the EMM superimposed with the EFCs for transverse and longitudinal waves in stainless steel at $43.8 \mathrm{kHz}$; b) divergence and c) curl of the velocity fields at $f_{\mathrm{c}}=43.8 \mathrm{kHz}$; taken from Zhu et al. [47]

To precisely explore refracted wave modes and the way of the incident longitudinal wave propagation through the EMM, the equi-frequency curves (EFCs) of the metamaterial in the frequency range of $39 \mathrm{kHz}$ to $44.6 \mathrm{kHz}$ in the first Brillouin zone are plotted in
Fig. 12a. The wave refraction is examined at the frequency $f_{\mathrm{c}}=43.8 \mathrm{kHz}$, in which both the NMD and NBM are present, and the EFC for this frequency is highlighted by red solid curve in the contour. In addition, the EFCs for the $\mathrm{P}$ and $\mathrm{S}$ wave in the host stainless steel at the same frequency are also plotted in the figure as the blue dotted circle and green dashed circle, respectively. In the figure, $\mathbf{V}_{\mathrm{g}}$ and $\mathbf{k}$ are the group velocity and wave vector of the incidental wave in the metamaterial, respectively, and $\mathbf{V}_{\mathrm{gt}}$ is the group velocity of S wave in the stainless steel. The refracted wave can be determined in the EFCs by using the Snell's law, which states that the components of the wave vector parallel to the refracting interface have to be the same for the incident and refracted waves. Thus the wave vector of the refracted wave can be determined by drawing a line (black dashed line) passing the end point of incident wave vector and being perpendicular to the interface, and then seeking the intersections of the line with the EFCs of the host media. It is seen from the figure that the intersection occurs in the EFC of S waved of the host material, and a refractive wave with angle $\varphi=-37^{\circ}$ is predicted, while no $\mathrm{P}$ wave is refracted since any intersection with blue circle is impossible. A wave mode conversion from $\mathrm{P}$ to $\mathrm{S}$ wave is found. It should be noted that the red EFC at this frequency does not possess exactly a circular shape, hence the vectors of the group velocity and phase velocity are not exactly antiparallel. In order to confirm these founding, full wave simulation is conducted, the divergence and curl of the velocity field obtained from finite element solution are shown in Fig. 12b and c to distinguish the $\mathrm{P}$ and $\mathrm{S}$ wave contents, respectively. It is seen that very weak refracted longitudinal wave field can be observed and much stronger refracted transverse wave field is found, as expected. The refraction angle estimated from the simulation, as shown in Fig. 12c, also agrees well with the prediction based on EFC.

The negative refraction was validated by experiment. Due to 2D feature of the problem, the plate-based wave testing technique was adopted. The wedge-shape EMM array $(326 \mathrm{~mm} \times 192 \mathrm{~mm}$ $\times 1.5 \mathrm{~mm}$ ) was fabricated in a thin stainless steel (Grade 304) plate $(3048 \mathrm{~mm} \times 1829 \mathrm{~mm} \times 1.5 \mathrm{~mm}$ ) by using a precision laser cutting technique, which is shown in Fig. 13. The zoomed-in views of the metamaterial at different scales are also shown in the figure. In the experiment, the dimension of the host plate is chosen to be sufficiently large in order to avoid unwanted reflected waves from the boundary. Since the interested frequency is very low $(<50 \mathrm{kHz})$, the lowest symmetric Lamb wave mode ( $\mathrm{S} 0)$ can be a 
good approximation for the in-plane wave behavior. An incident $\mathrm{P}$ wave is launched by symmetrically bonded piezoelectric patches on top and bottom of the plate. In the test, a steady wave excitation with single frequency is avoided since the absorbing boundary for a solid plate is difficult to realize, instead, a tone burst signal with narrow frequency band is chosen. The refracted in-plane waves are measured on the surface of the plate by a 3D laser scanning vibrometer (Polytec PSV-400-3D). The frequency -wavenumber filtering technique is also applied to remove undesired out-of-plane mode generated due to the inaccuracies in the fabrication and experiment setup.
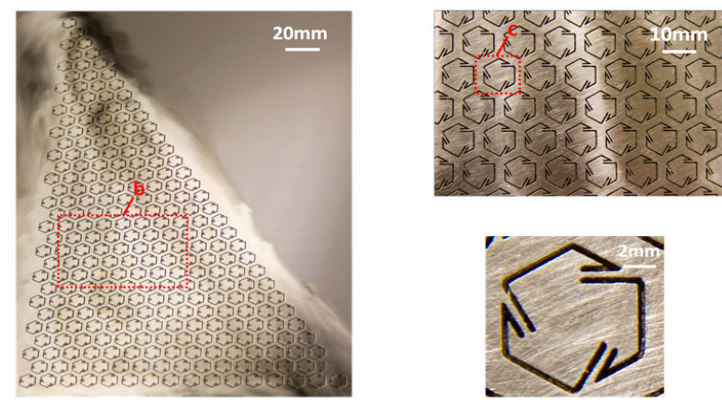

Fig. 13. The fabricated triangular array of the chiral EMM; taken

from Zhu et al. [47]

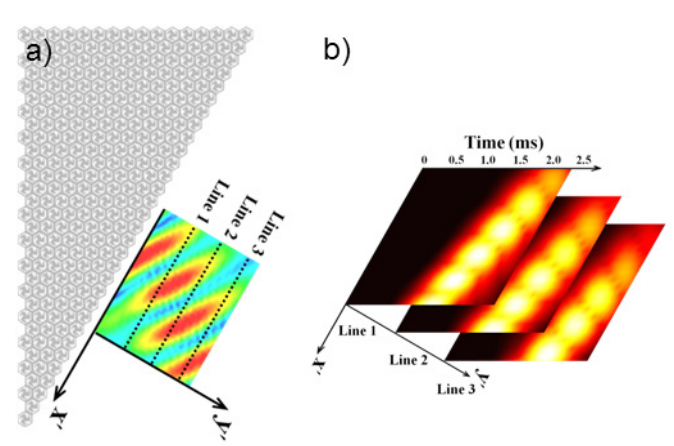

b)

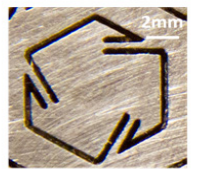

Fig. 14. a) The measured velocity field at $t=1.7 \mathrm{~ms}$ with frequency $43.8 \mathrm{kHz}$; b) Time domain signal envelops of the scanning points on the three selected lines; taken from Zhu et al. [47]

The snapshot of the measured amplitude of the in-plane velocity field for $t=1.7 \mathrm{~ms}$ at $43.8 \mathrm{kHz}$ is shown in Fig. 14. A local coordinate system $x^{\prime}-y^{\prime}$ is defined with $x^{\prime}$ parallel to the oblique interface. It can be clearly found that the measured refracted wave propagates downward in the negative refraction side with respective to the interface normal. The measured angle of the refraction is about $-38^{\circ}$, which is very close to the numerical prediction. Fig. 14b shows the amplitudes of the recorded wave signal envelopes in the time domain at three regularly spaced lines with a distance from each other by $33 \mathrm{~mm}$ along $y^{\prime}$ direction (see the inset in Fig. 14a) lines 1, 2 and 3). From this measurement the group velocity of the outgoing wave in the host material can be estimated as $V_{g}=3320 \mathrm{~m} / \mathrm{s}$, which is exactly the transverse wave speed of the steel plate as predicted previously.

\section{MICROPOLAR MODEL FOR CHARACTERIZING PLANAR CHIRAL LATTICES}

The effective property and wave characteristic of the chiral elastic metamaterial presented in the previous sections are considered in the framework of classical Cauchy elasticity, and this theory succeeds in material design and the interpretation of the observed wave phenomena. However, chiral material cannot be fully described by Cauchy elasticity since it is not able to characterize the handedness of the material [48]. For example, consider the tri-chiral lattice and its mirror reflection shown in Fig. 15, the difference between the two figures should be reflected somehow by constitutive equations. However, classical elastic theory remains the same upon a transformation of mirror reflection, which is equivalent to the inversion of one coordinate axis. It is interesting to note that, for the tri-chiral lattice, if we adopt the sign convention of topology parameter $\beta$ according to the relative orientation of the ligament and the link of circle centers, the sign of $\beta$ monitors the handedness of the lattice, as indicated in Fig. 15. This operation cannot be achieved by an in-plane rotation due to its chiral nature. To more comprehensively characterize the 2D chiral solids, one should head to the higher order elastic theory, e.g. micropolar theory. For 3D case, it is found early that isotropic chiral elastic material can be well characterized by the non-centrosymmetric micropolar theory [48]. The recently developed 2D chiral micropolar theory [49] to [51], and some new wave phenomena are reviewed in this section.
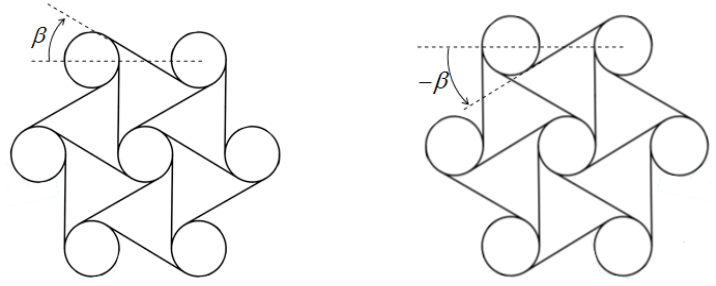

Fig. 15. Chiral lattice with a) $\beta>0$ and its handedness reversed pattern with b) $\beta<0$

Characterization of material chirality is closely related to the concept of pseudo (or axial) tensors which alternate sign under a mirror reflecting 
transformation or the handedness change of the underlying coordinate system, while ordinary (or polar) tensors are not affected by such actions. In micropolar theory, rotational degree of freedom (DOF) $\phi_{i}$ is introduced in addition to the displacement $\underline{u}_{i}$ at a material point [52]. The deformation measures are characterized as strain and curvature:

$$
\varepsilon_{k l}=u_{l, k}+e_{l k m} \phi_{m}, \quad \kappa_{k l}=\phi_{k, l}
$$

respectively, and the balances of stress $\sigma_{j i}$ and couple stress $m_{j i}$ are governed by:

$$
\sigma_{j i, j}=\rho \partial^{2} u_{i} / \partial t^{2}, \quad m_{j i, j}+e_{i k l} \sigma_{k l}=J \partial^{2} \phi_{i} / \partial t^{2},
$$

where $e_{i j k}$ is the Levi-Civita tensor, $\rho$ and $J$ are the density and micro-inertia, respectively. Subscripts of Latin letters range from 1 to 3, subscripts of Greek letters range from 1 to 2 , and a comma in subscript denotes partial differentiation with respect to coordinates. The governing equations is completed by the constitutive equations:

$$
\sigma_{i j}=C_{i j k l} \varepsilon_{k l}+B_{i j k l} \phi_{k, l}, \quad m_{i j}=B_{i j k l} \varepsilon_{k l}+D_{i j k l} \phi_{k, l},
$$

where $\mathbf{C}, \mathbf{D}$ and $\mathbf{B}$ are elastic tensors of rank four. Consider 2D problem defined in the $x_{1}-x_{2}$ plane where $u_{3}=\phi_{1}=\phi_{2}=\partial / \partial x_{3}=0$, Eqs. (20) and (21) reduce to:

$$
\begin{gathered}
\left\{\begin{array}{c}
\varepsilon_{\alpha \beta}=u_{\beta, \alpha}+e_{\beta \alpha} \phi \\
k_{\alpha}=\phi_{, \alpha}
\end{array}\right. \\
\left\{\begin{array}{c}
\sigma_{\beta \alpha, \beta}=\rho \partial^{2} u_{\alpha} / \partial t^{2} \\
m_{\alpha, \alpha}+e_{\alpha \beta} \sigma_{\alpha \beta}=J \partial^{2} \phi / \partial t^{2}
\end{array}\right.
\end{gathered}
$$

respectively, where $\phi_{3} \equiv \phi, \quad \kappa_{\alpha 3} \equiv \kappa_{\alpha}, \quad m_{\alpha 3} \equiv m_{\alpha}$ are defined for brevity, and $e_{\alpha \beta} \equiv e_{3 \alpha \beta}$ can be considered as the 2D Levi-Civita tensor. Liu et al. [49] proved that 2D isotropic chiral micropolar material should be characterized by the following constitutive equation which is for clarity written in a matrix form:

$$
\left\{\begin{array}{l}
\sigma_{11} \\
\sigma_{22} \\
\sigma_{12} \\
\sigma_{21} \\
m_{13} \\
m_{23}
\end{array}\right\}=\left[\begin{array}{cccccc}
2 \mu+\lambda & \lambda & -A & A & 0 & 0 \\
\lambda & 2 \mu+\lambda & -A & A & 0 & 0 \\
-A & -A & \mu+\kappa & \mu-\kappa & 0 & 0 \\
A & A & \mu-\kappa & \mu+\kappa & 0 & 0 \\
0 & 0 & 0 & 0 & \gamma & 0 \\
0 & 0 & 0 & 0 & 0 & \gamma
\end{array}\right]\left\{\begin{array}{c}
u_{1,1} \\
u_{2,2} \\
u_{2,1}-\phi \\
u_{1,2}+\phi \\
\phi_{, 1} \\
\phi_{, 2}
\end{array}\right\} .
$$

There are four classical micropolar elastic constants (Lame's constants $\lambda$ and $\mu$, antisymmetric shear modulus $\kappa$ and higher order modulus $\gamma$ ) and a new parameter $A$ characterizing the chiral effect. When the handedness of the material pattern is reversed, the chiral constant $A$ should reverse its sign, and the other constants remain unchanged. From Eqs. (23) to (25), the wave equations of 2D isotropic chiral micropolar material read:

$$
\begin{aligned}
\rho \frac{\partial^{2} u}{\partial t^{2}}= & (\lambda+2 \mu) u_{, x x}+(\mu+\kappa) u_{, y y}+(\lambda+\mu-\kappa) v_{, x y}+ \\
& +2 \kappa \phi_{, y}-A\left(v_{, x x}-v_{, y y}-2 u_{, x y}-2 \phi_{, x}\right), \\
\rho \frac{\partial^{2} v}{\partial t^{2}}= & (\lambda+2 \mu) u_{, x x}+(\mu+\kappa) u_{, y y}+(\lambda+\mu-\kappa) v_{, x y}+ \\
& +2 \kappa \phi_{, y}-A\left(v_{, x x}-v_{, y y}-2 u_{, x y}-2 \phi_{, x}\right), \\
J \frac{\partial^{2} \phi}{\partial t^{2}}= & \gamma\left(\phi_{, x x}+\phi_{, y y}\right)-4 \kappa \phi+2 \kappa\left(v_{, x}-u_{, y}\right)- \\
& -2 A\left(u_{, x}+v_{, y}\right) .
\end{aligned}
$$

The form of Eq. (26) looks like those of anisotropic medium, however, they are essentially different and cannot be covered by any anisotropy without chirality, since the parameter $A$ and its sign form a unique pattern in the constitutive matrix in Eq. (25). The positive definiteness of the strain energy density imposes condition $A^{2}<(\lambda+\mu) \kappa$ on the chiral constant $A$, which can be either positive or negative, yet its absolute value is bounded.

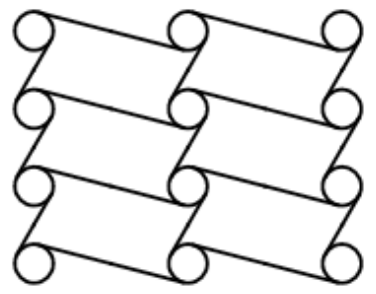

Fig. 16. Tetra-chiral lattice

For the tetra-chiral lattice shown in Fig. 16, obviously a 2D orthotropic chiral micropolar constitutive model is needed, which is recently given by Chen et al. [50] and [51] employing the theory of irreducible orthogonal tensor decomposition. The obtained constitutive tensors display a hierarchy structure depending on symmetry of underlying microstructure. Depending on the microstructure symmetry, up to eight material constants, in addition to the five for the isotropic case, are introduced to characterize a 2D orthotropic chiral elastic material.

The constitutive Eq. (25) provides a more sophisticated framework to characterize the tri-chiral lattice introduced in Section 2. By assuming the circle of the tri-chiral lattice is rigid, the five effective micropolar constants of the tri-chiral lattice can be analytically obtained as: 


$$
\begin{aligned}
& \lambda=\frac{\sqrt{3} E_{s}}{4} \eta\left(\cos ^{2} \beta-\eta^{2}\right) \sec ^{3} \beta \cos 2 \beta, \\
& \mu=\frac{\sqrt{3} E_{s}}{4} \eta\left(\cos ^{2} \beta+\eta^{2}\right) \sec ^{3} \beta, \\
& \kappa=\frac{\sqrt{3} E_{s}}{2} \eta\left(\sin ^{2} \beta+\eta^{2}\right) \sec \beta, \\
& \gamma=-\frac{E_{s} L^{2}}{4 \sqrt{3}} \eta\left(3 \sin ^{2} \beta+2 \eta^{2}\right) \sec \beta, \\
& A=\frac{\sqrt{3} E_{s}}{2} \eta\left(\eta^{2}-\cos ^{2} \beta\right) \sec \beta \tan \beta,
\end{aligned}
$$

where $\eta=t_{\mathrm{b}} / R$ indicates the slenderness ratio of the ligaments. It can be verified that when the chiral lattice is flipped over (sign of $\beta$ is reversed), $A$ changes its sign, while all the other parameter remains unchanged. When $\beta=0$ for a traditional triangular lattice (see Fig. 1b), $A$ vanishes as expected.

The chiral micropolar theory can reveal unique wave property in 2D chiral solids which cannot be predicted by traditional elasticity theory. The most pronounced difference between the chiral and non-chiral micropolar media is that for the later a non-dispersive longitudinal wave with velocity $c_{p}=[(\lambda+2 \mu) / \rho]^{1 / 2}$ can always be decoupled from the other two shear-rotation coupled waves. This is the characteristic of the non-chiral micropolar media, i.e. the microrotation is only coupled with shear but not with dilatation. In the chiral micropolar theory, the rotation is coupled with the dilation deformation due to the non-zero chiral constant $A$. Hence there would be no longer pure $\mathrm{P}$ or pure $\mathrm{S}$ waves in such media. The three wave modes are all mixed and dispersive, thus we call P, S or R (rotation) dominated waves, respectively. Moreover, the common feature of circular polarization for 3D isotropic chiral micropolar material [53] is not presented in the current 2D case, i.e. material particles are linearly polarized during the wave motion. However, the loss of mirror symmetry is reflected in another way for the 2D case. Since the medium is in-plane isotropic, the frequency dispersion has to be isotropic and the EFCs of this medium should be concentric circles. On the other hand, the polarization will remain a fixed angle with respect to the wave vector. This feature is schematically shown in Fig. $17 \mathrm{a}$, where the polarization of mixed $\mathrm{P} / \mathrm{S}$ wave mode forms a chiral pattern without reflective symmetry. $\mathrm{P} / \mathrm{S}$ mixed polarization accompanied by isotropic dispersion (EFC) is a unique behavior which cannot be predicted by traditional elasticity theory.

Fig. 17b shows the dispersion curves (solid lines) of the tri-chiral lattice predicted by using the homogenized wave equation Eq. (26) in which the effective material constants Eq. (27) are taken. The lattice geometry is specified by $R=1.0, \beta=0.9$, $\eta=1 / 20$. The ligaments of the lattice are assumed to be massless, while the mass and rotational inertia of the rigid circles are assumed to be unit. The exact solution of the dispersion curves (circles) obtained from Bloch wave analysis of the tri-chiral lattice is also
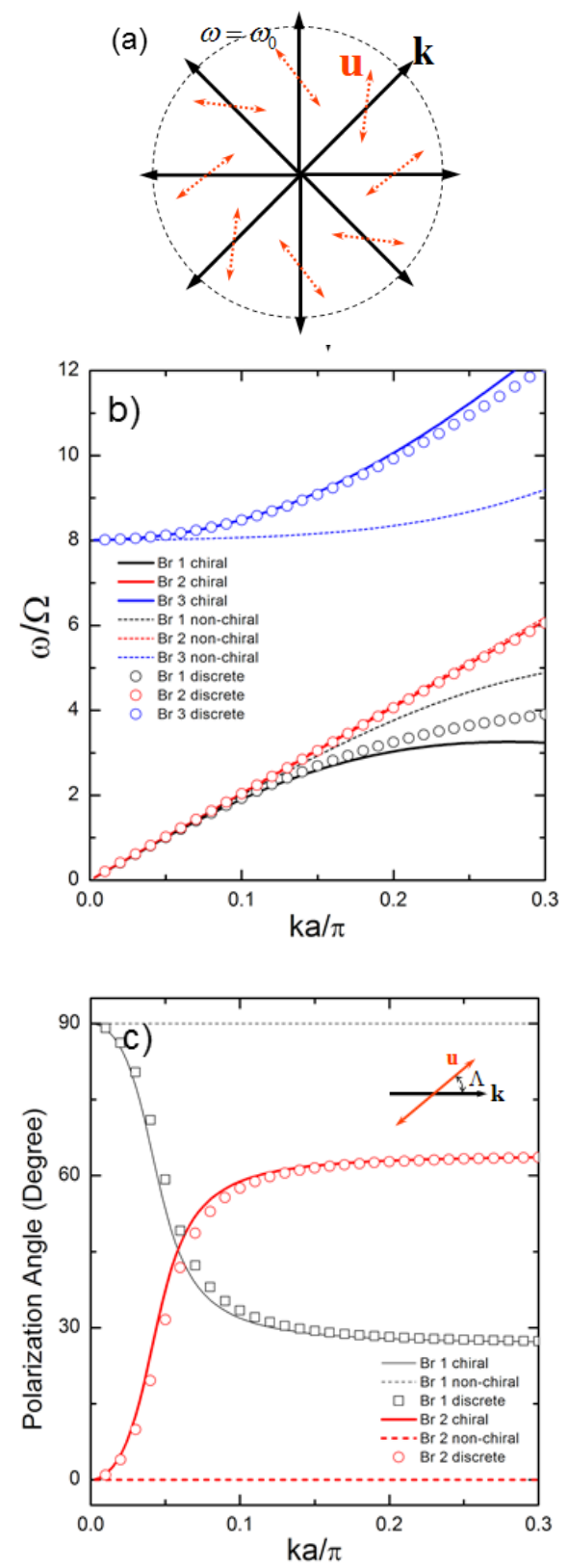

Fig. 17. a) Schematic wave behavior of the $2 D$ isotropic chiral material; comparison of b) dispersion curves and c) polarization angle for the chiral and non-chiral homogenization with the discrete model; taken from Liu et al. [49] 
plotted in the figure as a benchmark. For comparison, dispersion curves (dashed lines) predicted by a nonchiral version of micropolar homogenization [39] are also plotted in the figure. The dispersion curves are grouped in black, red and blue colors for the first, the second and the third branches, respectively. The first and second branches correspond to the displacement dominated modes, while the third one is the rotational dominated wave. The second branch is almost nondispersive. All three models agree well for this branch. However, for the first and third branches, the chiral theory agrees well with those given by the exact solution of the corresponding discrete model and a large discrepancy is found for the non-chiral theory. It is also interesting to notice that the dispersion curves of $\mathrm{P}$ and $\mathrm{S}$ dominated wave almost coincide at the long wave limit, indicating almost same phase wave velocities. This is the feature of waves in materials with Poisson's ratio $v=-1$, where the shear modulus is much greater than the bulk modulus.

Since the wave is linearly polarized, it is appropriate to examine the wave mode of the displacement dominated wave through the polarization angle $\Lambda$. The polarization angle of the first two branches predicted by the chiral and nonchiral theories are plotted in Fig. $17 \mathrm{c}$ as function of the wave number, where the first and second branches are marked in black and red, respectively. The non-chiral theory always predicts pure $\mathrm{P}$ and $\mathrm{S}$ waves, thus the polarization angles remain to be 0 and 90 degrees, as expected. For the chiral theory, the $\mathrm{S}$ and $\mathrm{P}$ dominated wave (for example $\Lambda>60^{\circ}$ and $\Lambda<30^{\circ}$ ) can be observed when the wave number is small for the first and second branches, respectively. However, for the intermediate wave number, the $\mathrm{P} / \mathrm{S}$ domination of the two branches become indistinguishable and can even interchange, i.e. the first branch become P-dominated and the second branch become S-dominated. Good agreement between the chiral micropolar homogenization and the exact discrete model (shown by circle and square dots) is found.

More recently, based on the chiral micropolar homogenization of the tri-chiral lattice and tetra-chiral lattice, Bacigalupo and Gambarotta [54], reconsidered the single- negative EMM lattice presented in Section 2. By adopting the matrix chiral lattice as a chiral micropolar continuum and considering the resonator as additional degree of freedoms which interact with each material point through appropriate translational and rotational stiffness, a generalized chiral micropolar continuum model with six degree of freedoms is established for the EMM lattice. The dynamic equations of the proposed model are

$$
\begin{gathered}
\left\{\begin{array}{c}
\sigma_{11,1}+\sigma_{12,2}+\hat{k}_{d}\left(v_{1}-u_{1}\right)=\rho_{1} \ddot{u}_{1} \\
\sigma_{21,1}+\sigma_{22,2}+\hat{k}_{d}\left(v_{2}-u_{2}\right)=\rho_{1} \ddot{u}_{2} \\
m_{1,1}+m_{2,2}+\sigma_{21}-\sigma_{12}+\hat{k}_{\theta}(\theta-\phi)=J_{1} \ddot{\phi}
\end{array}\right. \\
\left\{\begin{array}{l}
\hat{k}_{d}\left(u_{1}-v_{1}\right)=\rho_{2} \ddot{v}_{1} \\
\hat{k}_{d}\left(u_{2}-v_{2}\right)=\rho_{2} \ddot{v}_{2}, \\
\hat{k}_{\theta}(\theta-\phi)=J_{2} \ddot{\theta}
\end{array}\right.
\end{gathered}
$$

where $\left(u_{\alpha}, \phi\right)$ and $\left(v_{\alpha}, \theta\right)$ are the displacement and rotation of the host continuum and resonators, respectively, $\hat{k}_{d}$ and $\hat{k}_{\theta}$ are the translational and rotational stiffness derived from the property of the soft coating of the resonator. Constitutive equation and homogenized material constants are taken from Eqs. (25) and (27). With this formulation, the chiral EMM lattice presented in Section 2 can be analytically studied and optimized for the purpose of tuning bandgaps or even emphasizing the branch of negative group velocity.

\section{CONCLUSIONS}

Though a variety of mechanisms achieving EMM have been proposed for a long time, design of practical EMM with combined exotic property is still a difficult task due to complicated nature of elastic wave. In this paper we show that coupled rotation and bulk deformation of $2 \mathrm{D}$ chiral solids provides a new resonant mechanism for the design of EMMs, and related works are reviewed. With the help of the chirality and coupled resonances, double-negative EMM microstructure can be significantly simplified for fabrication. Moreover, the highly designable feature of chiral solids can also be explored to enhance the function of EMMs. On the other hand, the introduction of chirality needs more sophisticated continuum theory, e.g. micropolar theory, to better understand and characterize this material. We hope related research can promote the application of EMM on the advanced wave control.

\section{ACKNOWLEDGEMENTS}

This work was supported in part by National Natural Science Foundation of China under Grants Nos. 10972036, 11472044, 11521062, 11372035. 


\section{REFERENCES}

[1] Pendry, J.B., Holden, A.J., Stewart, W.J., Youngs, I. (1996). Extremely low frequency plasmons in metallic mesostructures. Physical Review Letters, vol. 76, no. 25, p. 4773-4776, D0I:10.1103/PhysRevLett.76.4773.

[2] Pendry, J.B., Holden, A.J., Robbins, D.J., Stewart, W.J. (1999). Magnetism from conductors and enhanced nonlinear phenomena. IEEE Transactions on Microwave Theory and Techniques, vol. 47, no. 11, p. 2075-2084, DOI:10.1109/22.798002.

[3] Veselago, V.G. (1968). The electrodynamics of substances with simultaneously negative values of $\varepsilon$ and $\mu$. Soviet Physics Uspekhi, vol. 10, no. 4, p. 509-514, Dol:10.1070/ PU1968v010n04ABEH003699.

[4] Shelby, R.A., Smith, D.R., Schultz, S. (2001). Experimental verification of a negative index of refraction. Science, vol. 292, no. 5514, p. 77-79, D0I:10.1126/science.1058847.

[5] Pendry, J.B., Schurig, D., Smith, D.R. (2006). Controlling electromagnetic fields. Science, vol. 312, no. 5781, p. 17801782, D0I:10.1126/science.1125907.

[6] Leonhardt, U. (2006). Optical conformal mapping. Science, vol. 312, no. 5781, p. 1777-1780, D0I:10.1126/science.1126493.

[7] Pendry, J.B. (2000). Negative refraction makes a perfect lens. Physical Review Letters, vol. 85, no. 18, p. 3966-3969, DOI:10.1103/PhysRevLett.85.3966.

[8] Schurig, D., Mock, J.J., Justice, B.J., Cummer, S.A., Pendry, J.B., Starr, A.F., Smith, D.R. (2006). Metamaterial electromagnetic cloak at microwave frequencies. Science, vol. 314, no. 5801, p. 977-980, DOl:10.1126/science.1133628.

[9] Liu, Z., Zhang, X., Mao, Y., Zhu, Y.Y., Yang, Z., Chan, C.T., Sheng, P. (2000). Locally resonant sonic materials. Science, vol. 289, no. 5485, p. 1734-1736, Dol:10.1126/ science.289.5485.1734.

[10] Liu, Z., Chan, C.T., Sheng, P. (2005). Analytic model of phononic crystals with local resonances. Physical Review $B$, vol. 71, no. 1, p. 014103, D0l:10.1103/PhysRevB.71.014103.

[11] Milton, G.W., Willis, J.R. (2007). On modifications of Newton's second low and linear continuum elastodynamics. Proceedings of the Royal Society A, vol. 363, no. 463, p. 855880, DOI:10.1098/rspa.2006.1795.

[12] Yao, S., Zhou, X., Hu, G. (2010). Investigation of the negativemass behaviors occurring below a cut-off frequency. New Journal of Physics, vol. 12, p. 103025, D0l:10.1088/13672630/12/10/103025.

[13] Yang, Z., Mei, J., Yang, M., Chan, N.H., Sheng, P. (2008). Membrane-type acoustic metamaterial with negative dynamic mass. Physical Review Letters, vol. 101, no. 20, p. 204301, D0I:10.1103/PhysRevLett.101.204301.

[14] Mei, J., Ma, G., Yang, M., Yang, Z., Wen, W., Sheng, P. (2012). Dark acoustic metamaterials as super absorbers for lowfrequency sound. Nature Communications, vol. 3, art. no. 756, DOl:10.1038/ncomms1758.

[15] Yang, Z., Dai, H.M., Chan, N.H., Ma, G.C., Sheng, P. (2010). Acoustic metamaterial panels for sound attenuation in the 50-1000 Hz regime. Applied Physics Letters, vol. 96, p. 041906, D0I:10.1063/1.3299007.
[16] Huang, H.H., Sun, C.T. (2011). Locally resonant acoustic metamaterials with 2D anisotropic effective mass density. Philosophical Magazine, vol. 91, no. 6, p. 981-996, Dol:10.10 80/14786435.2010.536174.

[17] Liu, A.P., Zhu, R., Liu, X.N., Hu, G.K., Huang, G.L. (2012). Multi-displacement microstructure continuum modeling of anisotropic elastic metamaterials. Wave Motion, vol. 49, no. 3 , p. 411-426, DOI:10.1016/j.wavemoti.2011.12.006.

[18] Zhu, R., Liu, X.N., Huang, G.L., Huang, H.H., Sun, C.T. (2012). Microstructural design and experimental validation of elastic metamaterial plates with anisotropic mass density. Physical Review B, vol. 86, p. 144307, D0l:10.1103/ PhysRevB.86.144307.

[19] Torrent, D., Sanchez-Dehesa, J. (2008). Anisotropic mass density by two-dimensional acoustic metamaterial. New Journal of Physics, vol. 10, p. 023004, D0l:10.1088/13672630/10/2/023004.

[20] Zigoneanu, L., Popa, B.-I., Starr, A.F., Cummer, S.A. (2011). Design and measurements of a broadband two-dimensional acoustic metamaterial with anisotropic effective mass density. Journal of Applied Physics, vol. 109, p. 054906, DOl:10.1063/1.3552990.

[21] Fang, N., Xi, D., Xu, J., Ambati, M., Srituravanich, W., Sun, C., Zhang, X. (2006). Ultrasonic metamaterials with negative modulus. Nature Materials, vol. 5, p. 452-456, D0I:10.1038/ nmat1644.

[22] Wu, Y., Lai, Y., Zhang, Z.-Q. (2007). Effective medium theory for elastic metamaterials in two dimensions. Physical Review B, vol. 76, no. 20, p. 205313, D0l:10.1103/PhysRevB.76.205313.

[23] Zhou, X., Hu, G. (2009). Analytic model of elastic metamaterials with local resonances. Physical Review B, vol. 79, p. 195109, Dol:10.1103/PhysRevB.79.195109.

[24] Ding, Y., Liu, Z., Qiu, C., Shi, J. (2007). Metamaterial with simultaneously negative bulk modulus and mass density. Physical Review Letters, vol. 99, no. 9, p. 093904, D0I:10.1103/PhysRevLett.99.093904.

[25] Li, J., Chan, C.T. (2004). Double-negative acoustic metamaterial. Physical Review E, vol. 70, no. 5, p. 055602 , D0l:10.1103/PhysRevE.70.055602.

[26] Deng, K., Ding, Y., He, Z., Zhao, H., Shi, J., Liu, Z. (2009). Theoretical study of subwavelength imaging by acoustic metamaterial slabs. Journal of Applied Physics, vol. 105, p. 124909, DOI:10.1063/1.3153976.

[27] Wu, Y., Lai, Y., Zhang, Z.-Q. (2011). Elastic metamaterials with simultaneously negative effective shear modulus and mass density. Physical Review Letters, vol. 107, no. 10, p. 105506 , D0l:10.1103/PhysRevLett.107.105506.

[28] Lai, Y., Wu, Y., Sheng, P., Zhang, Z.-Q. (2011). Hybrid elastic solids. Nature Materials, vol. 10, p. 620-624, D0l:10.1038/ nmat3043.

[29] Zhang, S., Yin, L., Fang, N. (2009). Focusing ultrasound with an acoustic metamaterial network. Physical Review Letters, vol. 102, no. 19, p. 194301, Dol:10.1103/ PhysRevLett.102.194301.

[30] Zhang, S., Xia, C., Fang, N. (2011). Broadband acoustic cloak for ultrasound waves. Physical Review Letters, vol. 106, no. 2, p. 024301, D0I:10.1103/PhysRevLett.106.024301. 
[31] Bongard, F., Lissek, H., Mosig, J.R. (2010). Acoustic transmission line metamaterial with negative/zero/positive refractive index. Physical Review B, vol. 82, no. 9, p. 094306 , DOl:10.1103/PhysRevB.82.094306.

[32] Oh, J.H., Seung, H.M., Kim, Y.Y. (2014). A truly hyperbolic elastic metamaterial lens. Applied Physics Letters, vol. 104, p. 073503, DOI:10.1063/1.4865907.

[33] Farhat, M., Guenneau, S., Enoch, S. (2009). Ultrabroadband elastic cloaking in thin plates. Physical Review Letters, vol. 103, no. 2, p. 024301, D0l:10.1103/PhysRevLett.103.024301.

[34] Finocchio, G., Casablanca, O., Ricciardi, G., Alibrandi, U., Garescì, F., Chiappini, M., Azzerboni, B. (2014). Seismic metamaterials based on isochronous mechanical oscillators. Applied Physics Letters, vol. 104, p. 191903, DOI:10.1063/1.4876961.

[35] Kelvin, W. (1894). The Molecular Tactics of a Crystal. Clarendon Press, Oxford.

[36] Pendry, J.B. (2004). A chiral route to negative refraction. Science, vol. 306, no. 5700, p. 1353-1355, Dol:10.1126/ science.1104467.

[37] Chen, H., Fung, K.H., Ma, H., Chan, C.T. (2008). Polarization gaps and negative group velocity in chiral phononic crystals: layer multiple scattering method. Physical Review B, vol. 77, p. 224304, D0I:10.1103/PhysRevB.77.224304.

[38] Spadoni, A., Ruzzene, M. (2012). Elasto-static micropolar behavior of a chiral auxetic lattice. Journal of the Mechanics and Physics of Solids, vol. 60, no. 1, p. 156-171, DOI:10.1016/j.jmps.2011.09.012.

[39] Spadoni, A., Ruzzene, M., Gonella, S., Scarpa, F. (2009). Phononic properties of hexagonal chiral lattices. Wave Motion, vol. 46, no.7, p. 435-450, Dol:10.1016/j. wavemoti.2009.04.002.

[40] Prall, D., Lakes, R.S. (1997). Properties of a chiral honeycomb with a Poisson's ratio approximates -1. International Journal of Mechanical Sciences, vol. 39, no. 3, p. 305-314, D0l:10.1016/ S0020-7403(96)00025-2.

[41] Liu, X.N., Hu, G.K., Sun, C.T., Huang, G.L. (2011). Wave propagation characterization and design of two-dimensional elastic chiral metacomposite. Journal of Sound and Vibration, vol. 330, no. 11, p. 2536-2553, D0l:10.1016/j. jsv.2010.12.014.

[42] Zhu, R., Liu, X.N., Hu, G.K., Sun, C.T., Huang, G.L. (2014). A chiral elastic metamaterial beam for broadband vibration suppression. Journal of Sound and Vibration, vol. 333, no. 10, p. 2759-2773, DOl:10.1016/j.jsv.2014.01.009.

[43] Liu, X.N., Hu, G.K., Huang, G.L., Sun, C.T. (2011). An elastic metamaterial with simultaneously negative mass density and bulk modulus. Applied Physics Letters, vol. 98, p. 251907, DOI:10.1063/1.3597651.

[44] Wang, X. (2014). Dynamic behavior of a metamaterial system with negative mass and modulus. International Journal of Solids and Structures, vol. 51, no. 7-8. p. 1534-1541, D0I:10.1016/j.ijsolstr.2014.01.004.

[45] Li, Z., Wang, X. (2016). On the dynamic behavior of a twodimensional elastic metamaterial system. International Journal of Solids and Structures, vol. 78-79, p. 174-181, DOI:10.1016/j.jjsolstr.2015.08.028.

[46] Milton, G. (2002). The Theory of Composites. Cambridge University Press, Cambridge, D0l:10.1017/ cb09780511613357.

[47] Zhu, R., Liu, X.N., Hu, G.K., Sun, C.T, Huang, G.L. (2014). Negative refraction of elastic waves at the deepsubwavelength scale in a single-phase metamaterial. Nature Communications, vol. 5, art. no. 5510, D0l:10.1038/ ncomms6510.

[48] Lakes, R.S., Benedict, R.L. (1982). Noncentrosymmetry in micropolar elasticity. International Journal of Engineering Science, vol. 20, no. 10, p. 1161-1167, D0I:10.1016/00207225(82)90096-9.

[49] Liu, X.N., Huang, G.L., Hu, G.K. (2012). Chiral effect in plane isotropic micropolar elasticity and its application to chiral lattices. Journal of the Mechanics and Physics of Solids, vol. 60, no. 11, p. 1907-1921, D0l:10.1016/j.jmps.2012.06.008.

[50] Chen, Y., Liu, X.N., Hu, G.K., Sun, Q.P., Zheng, Q.S. (2014). Micropolar continuum modelling of bi-dimensional tetrachiral lattices. Proceedings of the Royal Society A, vol. 470, no. 2165, p. 20130734, DOl:10.1098/rspa.2013.0734.

[51] Chen, Y., Liu, X., Hu, G. (2014). Micropolar modeling of planar orthotropic rectangular chiral lattices. Comptes Rendus Mécanique, vol. 342, no. 5, p. 273-283, D0l:10.1016/j. crme.2014.01.010.

[52] Eringen, A.C. (1999). Microcontinuum Field Theories I: Foundations and Solids. Springer, New York, D0I:10.1007/9781-4612-0555-5.

[53] Lakhtakia, A., Varadan, V.V., Varadan, V.K. (1988). Elastic wave propagation in noncentrosymmetric, isotropic media: dispersion and field equations. Journal of Applied Physics, vol. 63, p. 5246-5250, D0I:10.1063/1.340387.

[54] Bacigalupo, A., Gambarotta, L. (2016). Simplified modelling of chiral lattice materials with local resonators. International Journal of Solids and Structures, vol. 83, p. 126-141, DOI:10.1016/j.jjsolstr.2016.01.005. 